\title{
Engorda de toretes con una dieta integral de excretas frescas de cerdo, melaza y pasto Taiwan (Pennisetum purpureum).
}

\section{Artículo Original}

Carlos F. Aguilar-Pérez, Eduardo R. Valencia-Heredia, Jorge S. Santos-Flores.

Depto. de Nutrición, Facultad de Medicina Veterinaria y Zootecnia, Universidad Autónoma de Yucatán, Mérida, Yucatán, México.

\section{RESUMEN.}

Introducción. El objetivo del presente estudio fue evaluar el cambio de peso, consumo y conversión alimenticia de toretes estabulados, durante su adaptación y engorda, con una dieta integral de excretas frescas de cerdo, melaza y pasto Taiwán (Pennisetum purpureum), y una dieta convencional de balanceado comercial y Taiwán.

Material y métodos. Se utilizaron doce toretes cruzados (Bos taurus x Bos indicus) con peso vivo y edad promedio de $271.3 \pm 51.9 \mathrm{~kg}$ y $378 \pm 79$ días, respectivamente. Los animales se dividieron en dos grupos para ser engordados hasta su peso al mercado (450 kg) con una dieta integral de excretas frescas de cerdo (EFC) (45\%), melazaurea $(31 \%)$ y Taiwán (24\%) (Tratamiento Experimental,TE); y con una dieta convencional de balanceado comercial (40\%) y Taiwán $(60 \%)$ (Tratamiento Testigo,TT).

Resultados. El consumo voluntario de materia seca (CVMS) para TE fue alto desde la segunda semana del período de adaptación (2.8 kg/100 kg PV). Al término de este período (28 días), no se encontraron diferencias significativas $(p>0.05)$ entre tratamientos para ganancia diaria de peso (GDP): $0.982 \mathrm{~kg}$ para TT y $0.798 \mathrm{~kg}$ para TE, aunque el CVMS y la conversión alimenticia (CA) fueron ligeramente mejores para TT (7.56 vs 7.09 $\mathrm{kg}$ MS/animal/día y 7.62 vs $8.89 \mathrm{~kg} \mathrm{MS} / \mathrm{kg}$ de ganancia). La GDP durante el período de engorda fue similar en ambos tratamientos, 0.677 para TT y $0.628 \mathrm{~kg} /$ animal/día para TE $(\mathrm{p}>0.05)$. Aunque el CVMS fue ligeramente mayor para TT (9.74 vs $8.77 \mathrm{~kg}$ MS/animal/ día), la CA fue muy similar en ambos tratamientos (14.39 vs $13.96 \mathrm{~kg}$ MS/kg ganancia para TT y TE, respectivamente).

Conclusiones. Se concluye que con el uso de EFC en la engorda de toretes se pueden obtener ganancias de peso similares a las de algunas dietas convencionales. (Rev Biomed 2002; 13:94-99)

Palabras clave: Excretas de cerdo, toretes, adaptación, engorda, producción animal.

\section{SUMMARY.}

Fattening of steers fed on an integral diet of fresh swine excrement, molasses and Taiwan

Solicitud de sobretiros: M.C. Carlos F. Aguilar-Pérez, Depto. de Nutrición, Facultad de Medicina Veterinaria y Zootecnia, Universidad Autónoma de Yucatán, Apartado Postal 4 - 116 Itzimná, C.P. 97100. Mérida, Yucatán, México. Tel. (999) 9423200, Fax: 9423205.

Recibido el 2/Febrero/2001. Aceptado para publicación el 21/Junio/2001. 


\section{CF Aguilar-Pérez, ER Valencia-Heredia, JS Santos-Flores.}

\section{grass (Pennisetum purpureum).}

Introduction. The objective of this study was to evaluate the weight change, intake and feed efficiency of confined steers through the adaptation and fattening period using an integral diet of fresh swine excrement (FSE), molasses and Taiwan grass, compared with a conventional diet of comercial food (cereal basis) and Taiwan grass.

Material and methods. Twelve crossbred steers (Bos taurus $x$ Bos indicus) of $271.3 \pm 51.9 \mathrm{~kg}$ of body weight were divided in two homogeneous groups and fattened until reaching $450 \mathrm{~kg}$ liveweight one group was fed with an integral diet based on fresh swine excrement (45\%), molassesurea (31\%) and chopped Taiwan Grass (Pennisetum purpureum) (24\%) (Experimental Treatment, ET), and the other with a conventional diet using a balanced commercial feed (40\%) and chopped Taiwan grass (60\%) (Control Treatment, CT).

Results. Voluntary dry matter intake (VDMI) for ET was high from the second week of adaptation (2.8 kg/100 kg live-weight). No significant difference $(p>0.05)$ was found at the end of the adaptation period (28 days) for daily live weight gain (DLWG) between CT $(0.982 \mathrm{~kg})$ and ET $(0.798 \mathrm{~kg})$, although VDMI and feed conversion (FC) were slightly better for CT (7.56 vs $7.09 \mathrm{~kg}$ dry matter/head/day and 7.62 vs $8.89 \mathrm{~kg}$ dry matter/ $\mathrm{kg}$ weight gain). Animal performance during the fattening period was similar for both treatments, with DLWG of 0.677 for CT and $0.628 \mathrm{~kg} / \mathrm{head} /$ day for ET ( $p>0.05)$. However, although VDMI was slightly higher for CT (9.74 vs $8.77 \mathrm{~kg} \mathrm{DM}$ / head/day), FC was similar in both treatments (14.39 vs $13.96 \mathrm{~kg} \mathrm{DM} / \mathrm{kg}$ weight gain, for CT and ET, respectively).

Conclusions. It is concluded that using FSE in the fattening of confined steers, similar live weight gains can be obtained as those found on some conventional diets. (Rev Biomed 2002; 13:94-99)

Key words: Swine excrement, steers, adaptation, fattening, animal production.

\section{INTRODUCCIÓN.}

En Yucatán, México, el inventario porcino ha aumentando considerablemente debido al desarrollo reciente de grandes centros de producción, lo que ha ocasionado la generación de grandes volúmenes de excretas (1). Se considera que cada cerdo excreta entre 0.5 y $0.8 \mathrm{~kg}$ de heces y $2.1 \mathrm{~kg}$ de orina por cada $100 \mathrm{~kg}$ de peso (2). Entre los residuos animales, el del cerdo es de los más contaminantes, pues su alto contenido de material orgánico e inorgánico propicia su degradación microbiana después de su excreción, produciendo gases, olores y contaminación del aire y del suelo. Esto último es de suma importancia en Yucatán, si se considera el tipo de suelo altamente permeable y la escasa profundidad de los mantos acuíferos en esta zona del país (3).

El valor nutricional de las excretas de cerdo, en especial su elevado contenido de proteína cruda ( $24 \%$ en base seca), les confiere un gran potencial para la alimentación de rumiantes. Además, su uso contribuiría significativamente a solucionar el problema de la contaminación ambiental y reduciría los costos de alimentación, que en sistemas intensivos pueden representar cerca del $80 \%$ de los costos de producción (4).

La mayoría de la información en el país sobre el uso de excretas frescas de cerdo (EFC) en la alimentación de rumiantes se ha generado en los últimos años, y éstas se han utilizado en altos niveles $(5,6)$ sin haberse registrado alteraciones en la salud o en la calidad de las canales de los animales al sacrificio (7).

El presente estudio pretendió validar algunos de esos resultados bajo condiciones de campo. El objetivo fue evaluar el cambio de peso, consumo y conversión alimenticia de toretes estabulados, durante su adaptación y engorda con una dieta integral de excretas frescas de cerdo, melaza y pasto Taiwán, y una convencional de alimento balanceado comercial (a base de cereales) y pasto Taiwán. 
Engorda de toretes.

\section{MATERIAL Y MÉTODOS.}

El experimento se llevó a cabo en el área bovina de la Facultad de Medicina Veterinaria y Zootecnia de la Universidad Autónoma de Yucatán (FMVZ-UADY), localizada a $20^{\circ} 52^{\prime} 3.86$ " de latitud norte y a $89^{\circ} 37^{\prime} 20.05^{\prime}$ "de longitud oeste. El clima de la región es tropical subhúmedo, la temperatura media anual fluctúa entre $24.6 \mathrm{y}$ $27.7^{\circ} \mathrm{C}$ y la precipitación pluvial anual oscila entre 415 y $1290 \mathrm{~mm}$, distribuidos mayormente entre mayo y octubre (3).

Se utilizaron 12 toretes cruzados (Bos taurus $x$ Bos indicus) con edades y pesos promedio de $378 \pm 79$ días y $271.3 \pm 51.9 \mathrm{~kg}$, respectivamente, con los que se formaron dos grupos homogéneos (seis toretes por grupo) para probar los siguientes tratamientos (datos en base seca):

1) Tratamiento Experimental.- Dieta integral de excretas frescas de cerdo (EFC) (45\%), melazaurea al $3 \%(31 \%)$ y forraje de corte Taiwán (Pennisetum purpureum Var.Taiwán) (24\%).

2) Tratamiento Testigo.- Alimento balanceado comercial a base de cereales $(40 \%)$ y forraje de corte Taiwán $(60 \%)$.

Los animales de cada tratamiento se alojaron en un corral colectivo, donde disponían de un comedero lineal de concreto y agua limpia a voluntad; no se proporcionaron sales minerales.

Las EFC provenían de la sección de engorda de la granja porcícola de la FMVZ-UADY y se recolectaban diariamente, en forma manual, poco antes de preparar la dieta. La mezcla de melazaurea consistió en: $94 \%$ de melaza, $3 \%$ de urea y $3 \%$ de agua. Las EFC y la preparación de melazaurea se mezclaban manualmente antes de ser incorporadas al forraje, el cual se cortaba diariamente de una misma parcela, irrigada y fertilizada ( $200 \mathrm{~kg} \mathrm{~N} / \mathrm{ha} / \mathrm{año}$ ). El forraje era picado antes de ofrecerse a los animales, a un tamaño de partícula de 5-10 cm, para ambos tratamientos. El alimento utilizado en el tratamiento testigo tenía una presentación en polvo y, según análisis de garantía, contenía $88 \%$ de materia seca (MS) y $13 \%$ de proteína cruda (PC).

\section{Período de adaptación.}

El período de adaptación y aceptación de las dietas duró cuatro semanas, con base en los resultados de experimentos previos $(5,6)$. Las dietas a probar sustituyeron semanalmente el $25 \%$ de la dieta habitual (pasto y concentrado). Los ingredientes de la dieta experimental se mezclaban en una revolvedora mecánica.

Al iniciar el período de adaptación se incorporó al grupo experimental un torete adaptado previamente a una ración similar, como lo sugieren algunos autores (8).

\section{Período de engorda.}

La duración de este período estuvo determinado por el crecimiento de los animales, quienes salían de la prueba al alcanzar el peso al mercado $(450 \mathrm{~kg})$. Los ingredientes de la dieta experimental se servían por separado: primero el forraje picado y sobre éste, la mezcla de EFC y melaza/urea. La dieta testigo (alimento balanceado comercial y pasto Taiwán) también se proporcionaba por separado. Ambos tratamientos se suministraban una sola vez al día, a las 11:00 a.m.

La ganancia diaria de peso (GDP), fue estimada mediante pesajes (previo período de ayuno de 18 horas) individuales cada catorce días. El consumo voluntario de materia seca (CVMS) se estimó por corral, a través de la medición diaria del alimento rechazado. La conversión alimenticia fue el resultado de dividir el CVMS entre la GDP. Se tomaron muestras catorcenales de los ingredientes, dietas y rechazos y se analizaron para determinar la materia seca (MS) y proteína cruda (PC), según A.O.A.C (9).

Con los datos de ganancia diaria de peso se corrió una prueba de comparación de medias, utilizando el estadístico " $t$ " de Student (10), con una significancia de 0.05 . Los datos de consumo de materia seca y conversión alimenticia se reportan como promedios de grupo. 


\section{CF Aguilar-Pérez, ER Valencia-Heredia, JS Santos-Flores.}

\section{RESULTADOS.}

En el cuadro 1 se presentan los promedios de materia seca (MS) y proteína cruda (PC) de la dieta integral y de los sustratos muestreados, en el período de adaptación.

\section{Cuadro 1}

Materia seca y proteína cruda de las excretas frescas de cerdo (EFC), dieta integral y componentes de la dieta testigo, utilizados en el período de adaptación.

\begin{tabular}{llc}
\hline \multicolumn{1}{c}{ Sustrato } & MS (\%) & PC (\%) \\
\hline Excretas frescas de cerdo & 20.99 & 23.02 \\
Pennisetum purpureum & 35.94 & 5.1 \\
Dieta integral $^{*}$ & 35.78 & 16.18 \\
Alimento comercial & 87.4 & 13.43 \\
\hline
\end{tabular}

${ }^{*} \mathrm{EFC}+$ Melaza-urea $3 \%+P$. purpureum

MS=Materia seca; $P C=$ Proteína cruda

En el cuadro 2 se presentan los datos semanales de consumo para los dos tratamientos. En el cuadro 3 se reportan los datos del comportamiento animal en el período de adaptación, referentes al consumo de alimento, ganancia de peso y conversión alimenticia. Se observó que, aunque el consumo de materia seca (CMS) y la ganancia diaria de peso (GDP) fueron ligeramente mayores para el grupo testigo, las diferencias no fueron significativas ( $p>0.05)$. Por lo que respecta a la conversión alimenticia (CA), ésta tendió a ser favorable para los toretes con la dieta convencional.

\section{Cuadro 2}

Consumo de materia seca (kg/animal/día) durante el período de adaptación de toretes estabulados, con una dieta convencional ( $T$. Testigo) y una a base de excretas frescas de cerdo (T. Experimental).

\begin{tabular}{ccc}
\hline Semana & T. Testigo & T. Exptal \\
\hline 1 & 5.98 & 5.23 \\
2 & 7.98 & 7.88 \\
3 & 7.98 & 8.05 \\
4 & 8.46 & 7.18 \\
\hline
\end{tabular}

Debido a problemas de intoxicación por urea en dos toretes del tratamiento experimental, se decidió suprimir ésta de la dieta en el día 66 del período de engorda.

En el cuadro 4 se presentan los promedios de MS y PC de la dieta integral y de los sustratos muestreados durante el período de engorda.

La GDP fue similar $(p>0.05)$ en ambos tratamientos (cuadro 5). Aunque el consumo voluntario de MS fue mayor para el grupo testigo, la conversión alimenticia fue similar para ambas dietas.

\section{Cuadro 3}

Cambio de peso, consumo y conversión alimenticia de toretes estabulados, alimentados con una dieta comercial (T. Testigo) y una a base de excretas frescas de cerdo (EFC) (T. Experimental), en el período de adaptación (28 días).

\begin{tabular}{|c|c|c|}
\hline Concepto & T. Testigo & T.Experimental \\
\hline Peso inicial (kg) & $271 \pm * 58.2$ & $271 \pm 50.5$ \\
\hline Peso Final (kg) & $298 \pm 67$ & $294 \pm 48$ \\
\hline $\begin{array}{l}\text { Consumo voluntario } \\
\text { (kg MS/100 kg PV) }\end{array}$ & 2.63 & 2.51 \\
\hline $\begin{array}{l}\text { Consumo voluntario } \\
\text { (kg/animal/día) }\end{array}$ & 7.48 & 7.09 \\
\hline $\begin{array}{l}\text { Ganancia diaria de peso }(\mathrm{kg}) \dagger \\
\text { Conversión alimenticia }\end{array}$ & $0.982 \pm 0.342$ & $0.798 \pm 0.261$ \\
\hline (kg MS/kg ganancia de PV) & 7.62 & 8.89 \\
\hline
\end{tabular}

\section{DISCUSIÓN.}

La GDP obtenida con la dieta de EFC en el período de adaptación fue superior a la registrada en estudios previos, con toretes alimentados en forma similar. Al respecto, se han reportado ganancias de peso de apenas .019 kg/animal/día (11), o incluso pérdidas de hasta $4 \mathrm{~kg}$ por animal (2) al finalizar el período de adaptación. La mejor respuesta de los animales en el presente experimento estaría en relación directa con la más temprana ingestión de altas cantidades de materia seca, lo que en dietas a base de excretas porcinas

\section{Revista Biomédica}


Engorda de toretes.

es de gran importancia (8).

La dieta de EFC fue bien aceptada desde la segunda semana (cuadro 2). Considerando el peso inicial de los animales $(271 \mathrm{~kg})$, la cantidad de MS consumida representaría más del $2.8 \%$ del peso vivo. Este valor es superior a lo reportado por otros autores $(5,6)$, que registraron índices de consumo para las primeras semanas por debajo del $2 \%$ del peso vivo. Observaciones recientes han sugerido que la utilización de animales ya acostumbrados al consumo de raciones con EFC puede ayudar a reducir el período de adaptación (8).

\section{Cuadro 4}

Materia seca y proteína cruda de las dietas y sus componentes durante el período de engorda.

\begin{tabular}{llc}
\hline \multicolumn{1}{c}{ Sustrato } & MS (\%) & PC (\%) \\
\hline Excretas frescas de cerdo (EFC) & 22.4 & 22.95 \\
Pennisetum purpureum & 30.77 & 5.54 \\
Melaza & 75.57 & 1.83 \\
Dieta integral $^{*}$ & 29.85 & 15.97 \\
Alimento comercial & 89.06 & 13.74 \\
\hline
\end{tabular}

${ }^{*} \mathrm{EFC}+$ Melaza + P. purpureum

MS=Materia seca; $\mathrm{PC}=$ Proteína cruda

Los signos de intoxicación por urea en algunos animales del grupo experimental, sugerirían que en dietas con altas proporciones de excretas no es necesario añadir urea, considerando la gran cantidad de nitrógeno amoniacal del estiércol de cerdo, máxime si se usa fresco, ya que es cuando posee una elevada cantidad de orina $(2,12)$.

La respuesta de los toretes a la dieta con EFC coincidió con otros resultados (13), con una dieta integral de EFC (35\%), melaza (35\%) y pasto merkerón (Pennisetum purpureum) (30 \%). Estos autores obtuvieron en promedio una GDP de 0.625 $\mathrm{kg} / \mathrm{animal}$, en toretes cruzados, de $250 \mathrm{~kg}$ de peso vivo y 16 meses de edad. Sin embargo, en investigaciones con dietas integrales de EFC y melaza, pero con rastrojo de maíz como forraje, la GDP fue cercana a $0.900 \mathrm{~kg} /$ animal/día $(14,15)$ e incluso superior a $1.0 \mathrm{~kg} /$ animal/día $(5,6)$. La conversión alimenticia en esas investigaciones también fue mejor, $9.62 \mathrm{~kg}$ (5) y $7.85 \mathrm{~kg}$ (14), aunque el consumo de MS fue similar a lo observado en el presente experimento. Es posible que la mejor ganancia de peso y conversión alimenticia en esos experimentos se hayan debido al uso de animales más jóvenes, o bien, estabulados en forma individual $(4,16)$.

De acuerdo con los resultados, en dietas con altas proporciones de excretas frescas de cerdo es inconveniente e innecesaria la adición de urea, dado sus altos contenidos de nitrógeno no proteico. Además, es posible reducir el tiempo de aceptación de estas dietas, evitando la selectividad de los ingredientes, por medio del mezclado y con el empleo de un animal previamente adaptado.

Los resultados obtenidos en el presente experimento destacan el valor de las EFC como

Cuadro 5

Cambio de peso, consumo y conversión alimenticia de toretes estabulados, alimentados con una dieta comercial (T. Testigo) y una a base de excretas frescas de cerdo (EFC) (T. Experimental), en el período de engorda.

\begin{tabular}{lcc}
\hline Concepto & T. Control & T. Experimental \\
\hline Peso inicial (kg) & $298 \pm * 68$ & $305 \pm 59$ \\
Peso final (kg) & $457 \pm 11$ & $447 \pm 15$ \\
Consumo voluntario (kg MS/100 kg PV) & 2.58 & 2.33 \\
Consumo voluntario (kg/animal/día) & 9.74 & 8.77 \\
Ganancia diaria de peso (kg) $\dagger$ & $0.677 \pm 0.126$ & $0.628 \pm 0.133$ \\
Conversión alimenticia (kg MS/kg ganancia de PV) & 14.39 & 13.96 \\
Tiempo de engorda (días) & $247 \pm 101$ & $235 \pm 98$ \\
\hline
\end{tabular}

* $\pm=$ Desviación Estándar

$\dagger \mathrm{NS}(\mathrm{P}>0.05)$ 


\section{CF Aguilar-Pérez, ER Valencia-Heredia, JS Santos-Flores.}

alternativa para la engorda de bovinos y ponen de manifiesto que con su uso se pueden lograr ganancias de peso similares a las obtenidas con algunas dietas convencionales.

\section{REFERENCIAS.}

1.- $\mathrm{Ku}$ JC. Valor nutricional de la excreta porcina en la alimentación de los rumiantes. En: Memoria del Taller: Uso de la excreta de cerdo (cerdaza) en la alimentación de los rumiantes. FMVZ-UADY. Mérida, Yucatán. 1997; p. 57-67.

2.- Hennig A, Flachowsky G. Pig excrement as a new feedstuff for ruminants. Pig News and Information 1982. 3 (Pt3):269-74.

3.- Duch GJ. La conformación territorial del estado de Yucatán. UACH. Chapingo, Méx. México. 427 p.

4.- Preston TR, Willis ME. Producción Intensiva de Carne. México: Editorial Diana; 1974. p.736.

5.- Solorio SB. Comportamiento de bovinos alimentados con tres niveles de excretas frescas de cerdo $(35,45,55 \%$ B.S), melaza y rastrojo de maíz. Tesis Maestría en Producción Animal Tropical. FMVZ-UADY. Mérida; 1993. p. 109.

6.- Gutiérrez-Vázquez E, Peña-Pérez F, Partida-Partida M, Flachowsky G. Acceptance of fresh swine excreta by growing bulls. J Appl Anim 1994; 5:143-52.

7.- Piñón GE, Rentería SI, Gutiérrez VE. Aislamiento de microorganismos patógenos (aeróbicos) en estiércol fresco de cerdo antes y después de ser utilizado como integrante en la dieta de toretes. En: Memorias del I Encuentro Universitario de Investigación Científica, Tecnológica y Humanística. EMVZ-UMSNH. Morelia, Michoacán.1991; p. 9-12.

8.- Gutiérrez VE, Ku VJC. Utilización del estiércol de cerdo en la alimentación de los rumiantes. Nut AnimTrop 1996; 3 (Pt1):19-41.

9.- Association of Official Analytical Chemists (A O A C). Official Methods of Analysis (13 th ed.). Washington (DC); 1980.

10.- Steel RGD, Torrie JH. Bioestadística: Principios y procedimientos. 2a ed. Bogotá (Colombia): Editorial McGraw-Hill; 1988. P.622.

\section{Revista Biomédica}

11.- Gutiérrez VE, Peña PF. Adaptación de novillos alimentados con estiércol fresco de cerdo, melaza y rastrojo de maíz. En: Memorias del I Encuentro Interno sobre Investigación Veterinaria y Producción Animal. Morelia; EMVZ-UMSNH. 1990a; p. 19 -24.

12.- Yang Y, Liao CW, Hong CM, Chung. KT. Studies on the quantity and quality of swine wastes voided. In: Chung P. editors. Animal waste treatment and utilization. Proceedings of international symposium on biogas, microalgae \& livestock wastes. Council for Agricultural Planning and Development. Taipei, Taiwán; 1980. P. 303-15.

13.- Puerto NJL, Loría MMM. Utilización de excretas frescas de cerdo en la alimentación de rumiantes. En: FMVZ-UADY: Informe Anual de la Facultad de Medicina Veterinaria y Zootecnia, UADY. Mérida; 1991. p. 41-3.

14.- Gutiérrez VE, Peña PF.. Finalización de toretes alimentados con estiércol fresco de cerdo, melaza y rastrojo de maíz. In: Memorias del Primer Encuentro Interno sobre Investigación Veterinaria y Producción Animal. EMVZUMSNH, Morelia, Michoacán. 1990b; p. 25 -7.

15.- Velázquez MJO, Gutiérrez EV. Alimentación de toretes (Bos taurus y Bos indicus) en desarrollo con estiércol fresco de cerdo, melaza y rastrojo de maíz. En: Memorias de la $4^{\mathrm{a}}$ reunión sobre nutrición animal. FMVZ, UANL. Monterrey; Nuevo León. 1992. p. 19 -23.

16.- Shimada AS. Fundamentos de Nutrición Animal Comparativa. México: Consultores en producción animal, S.C.; 1983. P. 373. 